\title{
Late-State Ripening Dynamics of a Polymer/Clay Nanocomposite
}

\author{
Xiaoliang Wang, ${ }^{\dagger}$ Pingchuan Sun, ${ }^{*} *$ Gi Xue,,${ }^{\dagger}$ and H. Henning Winter $*, \S$ \\ ${ }^{\dagger}$ Department of Polymer Science and Engineering, Nanjing University, Nanjing 210093, China, ${ }^{\star}$ Key \\ Laboratory of Functional Polymer Materials, Ministry of Education, College of Chemistry, Nankai University, \\ Tianjin 300071, China, and ${ }^{\S}$ Department of Chemical Engineering and Department of Polymer Science and \\ Engineering, University of Massachusetts, Amherst, Massachusetts 01003
}

Received July 28, 2009; Revised Manuscript Received January 11, 2010

\begin{abstract}
A remarkably simple viscoelastic relaxation pattern was found for a physical gel that, beyond its gel point, slowly ripens toward a stable structural state. The material is a nanocomposite that consists of two components, an organoclay and an end-functionalized polymer, which get mixed at prescribed ratio. When freshly combined, the polymer intercalates into the clay galleries and eventually exfoliates the clay. The exfoliation occurs without applying flow. The nanocomposite quickly passes through the gel point and, with increasing ripening time, $t_{\mathrm{r}}$, its characteristic modulus $G_{\mathrm{c}}\left(t_{\mathrm{r}}\right)$ and relaxation time $\lambda_{\mathrm{c}}\left(t_{\mathrm{r}}\right)$ grow and decay by orders of magnitude, respectively. Surprisingly, their product, $G_{\mathrm{c}}\left(t_{\mathrm{r}}\right) \lambda_{\mathrm{c}}\left(t_{\mathrm{r}}\right)$, is found to remain constant during the two vastly different structuring processes of intercalation and exfoliation. For the rheological experiments, $G_{\mathrm{c}}\left(t_{\mathrm{r}}\right) \lambda_{\mathrm{c}}\left(t_{\mathrm{r}}\right)=$ constant means that dynamic mechanical data can be merged into each other by $\log / \log$ shifting under $45^{\circ}$.
\end{abstract}

\section{Introduction}

Nonequilibrium phenomena are pervasive in multicomponent, solid materials throughout the spectrum of materials applications, including pharmaceuticals, ion conductors for photovoltaic systems, personal care products, and gel-based drug delivery and tissue culture formulations. The internal structure of such man-made solid materials is rarely equilibrated, as they are not given sufficient time to ripen and/or the ripening process arrests before reaching equilibrium due to a free-energy landscape with multiple local minima. ${ }^{1-4}$ Nonequilibrium states determine not only the manufacturing and processing of these materials but also their end-use. The understanding of materials away from equilibrium has been recognized as one of the grand challenges to science and engineering. ${ }^{5}$ In spite of the importance of such outof-equilibrium materials, relatively little is known that could help to control the dynamics of structure development or tailor the structure for specific purposes. We therefore selected a model composite material with constituents that slowly connect into a disordered, three-dimensional structure and used rheology to study the evolving out-of-equilibrium states while approaching a stable end-state.

We use small-amplitude oscillatory shear to follow the growth of internal connectivity during the structural ripening process. The low-frequency dynamic moduli, $G^{\prime}(\omega, t)$ and $G^{\prime \prime}(\omega, t)$, are known to be especially sensitive measures of connectivity of internal structure. However, any data point on these curves can represent the ripening process as long as the $G^{\prime}$ and $G^{\prime \prime}$ curves maintain their general shape and can be shifted into a single pair of curves. Here we will represent the shifting through a characteristic modulus and a characteristic relaxation time

$$
G_{\mathrm{c}}(t)=\lim _{\operatorname{low} \omega} G^{\prime}(t) \quad \text { and } \quad \lambda_{\mathrm{c}}(t)=1 / \omega_{x}(t)
$$

\footnotetext{
*To whom correspondence should be addressed: Tel 1.413.5450922; Fax 1.413.5451647; e-mail winter@ecs.umass.edu.
}

During ripening in the solid state, the modulus grows and the relaxation time decays as shown schematically in Figure 1.

The characteristic modulus $G_{\mathrm{c}}$ denotes the low-frequency plateau value of $G^{\prime}$, which is a typical rheological property of ideal solids in the zero limit, $\omega \rightarrow 0$. Physical gels and soft glassy materials are not solids in this ideal sense since they can relax when given sufficient time which might be extremely long. However, physical gels behave as solids in a wide experimental time window, which includes a plateau modulus $G_{\mathrm{c}}$ at low frequencies. This is meant when taking the limit at "low $\omega$ " in eq 1 instead of taking the ultimate limit $\omega \rightarrow 0$. The relaxation at extremely long times is outside the experimental window of this study and will not be discussed here.

Throughout this study, the characteristic relaxation time, $\lambda_{\mathrm{c}}$, is defined as inverse of the crossover frequency, $\omega_{x}$, for which $G^{\prime}=$ $G^{\prime \prime}$, and is chosen because it is a distinct and measurable point on the modulus curves. A more meaningful choice as reference time would be the longest relaxation time if it were measurable unambiguously. For this study we prefer the crossover time as reference because of its accessibility.

The model system is thought to be representative for groups of physical gels and soft glasses that share the phenomenon of a slowly equilibrating structure when subjected to a jump in environment. ${ }^{6,7}$ Typically, the structure has not fully matured at the gel point and the long-time modulus keeps growing as shown in Figure 1. The longest relaxation time, which had diverged at the gel point, decays again as the structure continues to ripen. While much is known about the rheology at the gel point and its distinct power-law spectrum, ${ }^{8,9}$ no specific viscoelasticity pattern seems to be known for the evolving nonequilibrium states beyond the gel point. These late ripening stages are the focus of this study.

There have been indications that a general ripening pattern might exist beyond the gel point. Such expectation is based on the observation that similar master curves, through rescaling, represent the time-dependent rheology of such diverse materials as cross-linking epoxies, ${ }^{10}$ physical gelation of PVC plastisols, ${ }^{11}$ 


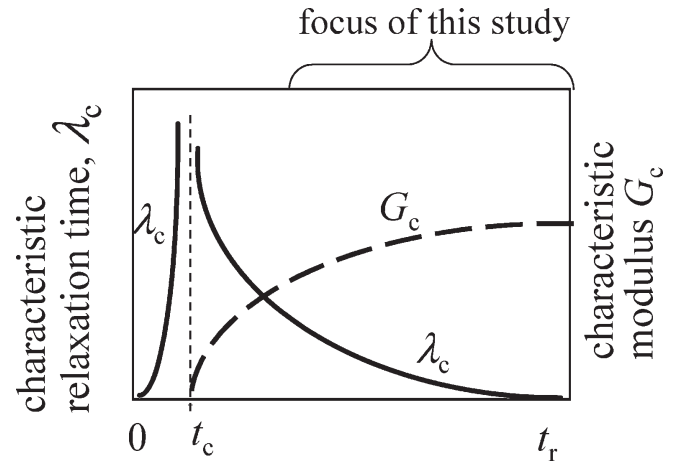

Figure 1. Rheology of physical gelation, schematically. Parameter is the ripening time, $t_{\mathrm{r}}$. Shown are a characteristic modulus after the gel point, $G_{\mathrm{c}}$, and a characteristic longest relaxation time, $\lambda_{\mathrm{c}}$, before and after $t_{\mathrm{c}}$ (see definition in eq 1). The early liquid-to-solid transition, marked by a diverging longest relaxation time at the gel point, $t_{\mathrm{c}}$, is fairly well understood. Most of the structural development in physical gels and soft glasses, however, occurs in the solid state where slow structural ripening dominates. This study addresses the question whether $G_{\mathrm{c}}$ and $\lambda_{\mathrm{c}}$ are related to each other.

aging of colloidal suspensions with weakly attractive particles, ${ }^{12}$ Laponite clay suspensions, ${ }^{13-18}$ and surface aggregation. This suggests that, when applying such scaling methods to our model material, a general framework of ripening dynamics might become apparent through the experimental findings with a model material.

We have recently reported a new class of gel-like telechelic polymer/exfoliated-clay nanocomposites using an end-functionalized polymer and highly anisotropic, organically modified silicates. ${ }^{19-22}$ Polymer/clay interactions not only enhance the clay dispersion, intercalation, and eventual exfoliation but also result in a sample spanning network structure. ${ }^{23-26}$ In the following, we will study the viscoelastic properties of the evolving structure of such a model physical gel with random clay orientation and rescale the data in search of a systematic rheological pattern beyond the gel point. We are mostly concerned with the time dependence of the rheological properties. The stable end-state (after a sufficiently long ripening period) serves as reference in the data analysis. Reproducibility is guaranteed by avoiding shearinduced orientation.

\section{Materials and Experimental Procedure}

Materials. The end-functionalized polymer is a dicarboxylterminated 1,4-polybutadiene oligomer (CTPB) with $M_{\mathrm{n}}=$ 4200 (Aldrich Chemical). CTPB is a fluid at room temperature which is advantageous for the performing the ripening experiments (no solvent needs to be added). The organoclay ( $\mathrm{C}_{18}$-clay) was prepared as follows: $10.0 \mathrm{~g}$ of industrially purified pristine montmorillonite (Tianjin Organic Clay Corp. China, cation exchange capacity is 1 mequiv/g) was dispersed into $1000 \mathrm{~mL}$ of distilled water at $87^{\circ} \mathrm{C}$ for cation exchange with $3.5 \mathrm{~g}$ (equal to 1 mequiv/g) of octadecyltrimethylammonium chloride (denoted as $\mathrm{C} 18 \mathrm{~A}$ ) for $12 \mathrm{~h}$. The processed clay was washed with distilled water and checked with a $0.1 \mathrm{~N} \mathrm{AgNO}_{3}$ solution for residual $\mathrm{Cl}^{-}$ ions. After the washing, the organoclay was dried at $87^{\circ} \mathrm{C}$ for $12 \mathrm{~h}$ before use. The process was described in detail elsewhere. ${ }^{19}$ The purpose of the C18A macrocounterions is to neutralize charge on the clay surfaces, to open the clay galleries, and to provide a thermodynamically compatible environment for the diffusion of the matrix molecules.

Sample Preparation. Organoclay/CTPB nanocomposites were prepared by gently and quickly mixing prescribed amounts of clay and CTPB in a disposable vial at ambient temperature $\left(15-20{ }^{\circ} \mathrm{C}\right)$ as described by Zhu et al. ${ }^{20}$ In our previous work we showed that no obvious orientation of clay sheets appeared during the mixing process. ${ }^{20,21}$ While maintaining quiescent conditions, the structure ripens into a stable clay dispersion as has be demonstrated previously. ${ }^{21}$ The ripening process begins after having mixed the clay into the CTPB $\left(\right.$ at $\left.t_{\mathrm{r}}=0\right)$. The freshly mixed sample undergoes a liquid-to-solid transition (physical gelation) very early since the initially aggregated clay particles already have sufficient surface area for the sticky matrix macromolecules to attach and to connect the tactoids into a samplespanning network structure. ${ }^{6}$ The early evolving relaxation pattern resembles gelation. ${ }^{8}$ However, its liquid-to-solid transition is only the beginning of the structural evolution. The molecules slowly swell the clay particles by diffusing into the clay galleries, a widely studied process that is called "intercalation". As further molecules keep diffusing into the galleries, the spacing of the clay sheets increases until they get so far apart that sheets cease to interact directly and begin to diffuse independently. This disintegration of the clay particles leads to a stable exfoliated clay (denoted as "SEC" in the following) structure with large-scale concentration fluctuations of randomly oriented, individual clay sheets throughout the polymer matrix. ${ }^{19-21}$ The model system naturally ripens into a SEC nanocomposite when heated up to a certain temperature $\left(>80^{\circ} \mathrm{C}\right)$. The existence of SEC as final, stable, and path-independent structure with unique rheology was confirmed experimentally for samples of this study. Exfoliation without applying shear flow is possible for a concentration of up to $10 \mathrm{wt} \%,{ }^{19-21}$ and we use $8 \mathrm{wt} \%$ clay in this study to ensure a final exfoliated structure. SEC might be close to equilibrium, but no attempt will be made here to find an ideal thermodynamic equilibrium.

Rheometry. The rheological measurements were performed on a strain-controlled rotational rheometer (ARES of TA Instruments Corp.). Cone-and-plate geometry of $50 \mathrm{~mm}$ diameter and 0.04 rad cone angle were chosen to ensure uniform shear throughout the samples. As measurement criterion, the strain amplitude was kept within the linear viscoelasticity region while being large enough for obtaining reasonable signal intensities in the frequency range $(0.01-100 \mathrm{rad} / \mathrm{s})$ and temperature range $\left(26-116^{\circ} \mathrm{C}\right)$. The temperature dependence of the dynamic storage modulus $\left(G^{\prime}\right)$ and loss modulus $\left(G^{\prime \prime}\right)$ was measured at frequencies $(\omega)$ of $1 \mathrm{rad} / \mathrm{s}$ and in the temperature range from 26 to $116^{\circ} \mathrm{C}$ with a heating rate of $2 \mathrm{~K} / \mathrm{min}$. Dynamic mechanical data during ripening were generated with a sequence of frequency sweeps between 100 and $0.2 \mathrm{rad} / \mathrm{s}^{8,27,28}$ and interpolated with the IRIS tools. ${ }^{29}$ To prevent the degradation of the samples, all the rheological experiments at elevated temperatures were performed under the protection of nitrogen gas.

Structure Characterization. TEM was carried out on a JEM 2100 electron microscope operated at $200 \mathrm{kV}$. Samples for TEM were cut into ultrathin sections with a diamond knife at a temperature of $-90{ }^{\circ} \mathrm{C}$ using a LKB2008 microtome. Microtomed thin sections were mounted on copper grids without staining. The TEM image (Figure 2) suggests that most of the clay sheets are disorderly dispersed in the polymer matrix after $12 \mathrm{~h}$ annealing at $80^{\circ} \mathrm{C}$.

\section{Results and Discussion}

Mechanical spectroscopy is able to measure the distribution of relaxation times and strengths of the material structure. The sample is exposed to small-amplitude oscillatory shear, $\dot{\gamma}(t)=\dot{\gamma}_{\mathrm{a}}$ $\sin (\omega t)$, while measuring the in-phase and out-of-phase contribution of the oscillating shear stress response, $\sigma(t)=G^{\prime} \dot{\gamma}_{\mathrm{a}} \sin (\omega t)+$ $G^{\prime \prime} \dot{\gamma}_{\mathrm{a}} \cos (\omega t)$, and hence defining the storage modulus $G^{\prime}$ and loss modulus $G^{\prime \prime} .{ }^{30}$ The period of the experiment, $t_{\mathrm{p}}=2 \pi /$ $\omega$, selectively probes relaxation processes with relaxation times in the order of $\tau \approx t_{\mathrm{p}}$. Large structural units require large relaxation times and vice versa. Correspondingly the long relaxation times of long-range connectivity in the gel will be especially visible in low frequency (i.e., long relaxation time) measurements. 


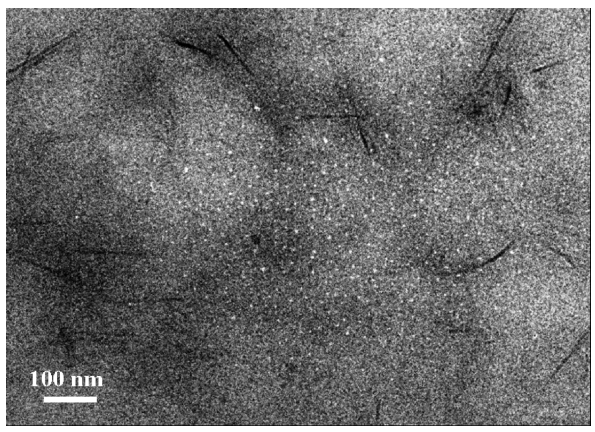

Figure 2. TEM of the $8 \mathrm{wt} \% \mathrm{C} 18 / \mathrm{CTPB}$ nanocomposite after having been annealed for $12 \mathrm{~h}$ at $80{ }^{\circ} \mathrm{C}$.

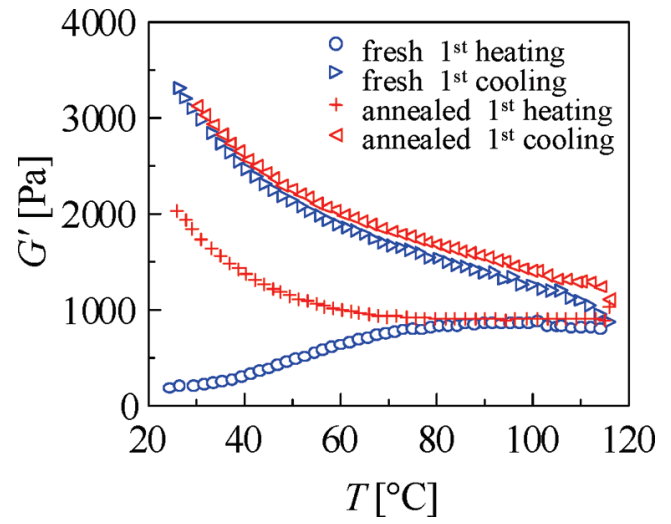

Figure 3. Temperature sweep of $\mathrm{CTPB} / \mathrm{C}_{18}$-clay $8 \mathrm{wt} \%$. Comparison of freshly mixed and annealed samples for $12 \mathrm{~h}$ at room temperature. $\omega=1 \mathrm{rad} / \mathrm{s}$, heating and cooling at $2 \mathrm{~K} / \mathrm{min}$. The samples were heated and then cooled. The annealed sample starts out with a partially developed structure.

Dynamic mechanical data during ripening were generated with a sequence of frequency sweeps between 100 and $0.2 \mathrm{rad} / \mathrm{s}$. Repetition of the multifrequency test ${ }^{31}$ while the sample is changing leads to an extensive $G^{\prime}, G^{\prime \prime}$ data set with each data point belonging to a new state of the material. Interpolation of the data results in "snapshots" $G^{\prime}\left(\omega, t_{\mathrm{r}}\right), G^{\prime \prime}\left(\omega, t_{\mathrm{r}}\right)$ of the material's viscoelasticity at distinct ripening times, $t_{\mathrm{r}}$. The IRIS software ${ }^{29}$ provides convenient and accurate interpolation of the timeresolved measurements. This time-resolved rheometry method ${ }^{27}$ was originally developed to study gelation, but it is ideally suited for an experimental study of out-of-equilibrium materials with time-dependent connectivity.

The experiments are in contrast to single frequency tests, which lack the information for a complete scaling analysis. The reason is the decay of the characteristic relaxation time scales of the evolving solid structure and the need for reducing the time scale of observation, $\omega^{-1}$, accordingly. Otherwise, each data point would belong to a new material-defined frequency, $\omega \lambda_{\mathrm{c}}\left(t_{\mathrm{r}}\right)$, and scaling would not be meaningful. Single-frequency scans, however, are useful for a quick test as will be shown next.

Accelerated Ripening at Elevated Temperature. A singlefrequency heating scan demonstrates the accelerated ripening at increased temperature (see Figure 3). A freshly mixed sample was compared to a sample that was allowed to slowly ripen at $26^{\circ} \mathrm{C}$ for $12 \mathrm{~h}$ after mixing. The storage modulus $\left(G^{\prime}\right)$ of the freshly mixed sample steadily increased upon heating from 26 to $80{ }^{\circ} \mathrm{C}$. $G^{\prime}$ increased further upon cooling with a final value that was 18 times its value before heating. $G^{\prime}$ of the $12 \mathrm{~h}$ annealed sample was already 11 times higher than $G^{\prime}$ of the freshly mixed sample (at $26{ }^{\circ} \mathrm{C}$ ). $G^{\prime}$ softened with increased temperature from 26 to $50{ }^{\circ} \mathrm{C}$ as expected form a

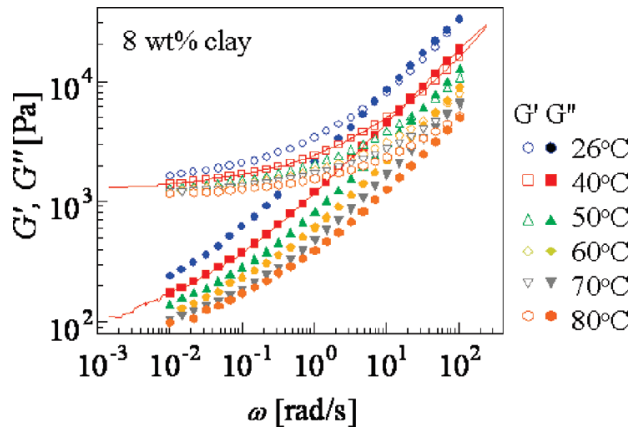

Figure 4. Temperature dependence of dynamic moduli of the stable exfoliated clay (SEC) structure that had been reached through heating to $120{ }^{\circ} \mathrm{C}$ for $20 \mathrm{~min}$. The continuous lines mark $G^{\prime}$ and $G^{\prime \prime}$ master curves at $40{ }^{\circ} \mathrm{C}$ (reference temperature for data in Figure 8).

polymeric material. When heated beyond $50^{\circ} \mathrm{C}$, its $G^{\prime}$ began to increase until it leveled off at about $80^{\circ} \mathrm{C}$. Both annealed and freshly mixed sample exhibit the same $G^{\prime}$ and $G^{\prime}$ after that heat treatment. These observations suggest that SEC with the same stable structure has established itself independently of the path of the ripening process.

Viscoelasticity of the Exfoliated State (SEC). $G^{\prime}(\omega)$ and $G^{\prime \prime}(\omega)$ of the SEC are shown in Figure 4. The $G^{\prime}$ master curve levels of at low frequencies. The $G^{\prime \prime}$ upturn of the low frequencies indicates the existence of a very slow relaxation mode as expected for a physical gel. However, this very slow mode is outside the experimental window (not shown here). The structure is stable over a wide temperature range and can be characterized rheologically with classical time-temperature superposition methods. The horizontal shift factor, $a_{\mathrm{T}}$, follows the typical WLF pattern, and the vertical shift, $b_{\mathrm{T}}$, can be expressed with Ferry's rubber elasticity term for polymers $\left(b_{\mathrm{T}}=\rho_{0} T_{0} / \rho T\right) .{ }^{30}$ With a temperature "scaling ratio"

$$
n_{\mathrm{T}}=\left(\log b_{\mathrm{T}}\right) /\left(\log a_{\mathrm{T}}\right) \approx 0
$$

the temperature shift for the SEC is horizontal. The welldefined reference state provides the framework for describing the rheological consequences of the evolving connectivity. $G^{\prime}$ and $G^{\prime \prime}$ during ripening can now be scaled by shifting to the corresponding SEC moduli. The limiting values of the characteristic relaxation time, $\lambda_{\mathrm{c}, \infty}(T)=\lim _{t_{\mathrm{r}} \rightarrow \infty} \lambda_{\mathrm{c}}\left(t_{\mathrm{r}}, T\right)$, and the low-frequency modulus, $G_{\mathrm{c}, \infty}(T)=\lim _{t_{\mathrm{r}} \rightarrow \infty} G_{\mathrm{c}}\left(t_{\mathrm{r}}, T\right)$, serve as scaling parameters.

Time-Resolved Rheometry of Ripening Dynamics. Timeresolved dynamic mechanical data at $T=40{ }^{\circ} \mathrm{C}$ and $T=$ $80^{\circ} \mathrm{C}$ are shown in Figure 5. Equivalent data were obtained at $T=26,50,60$, and $70{ }^{\circ} \mathrm{C}$. Interpolation of these data leads to the "snapshots" in Figure 6. At all temperatures of this study, the transition from liquid to solid occurred nearly instantaneously $\left(\sim 100 \mathrm{~s}\right.$ at $\left.40^{\circ} \mathrm{C}\right)$ so that most of the ripening happened beyond the gel point in the solid state. The typical solid behavior can be recognized by a tan $\delta$ (at low frequencies) which increases with frequency $\omega$ (not shown here).

Scaling of the Ripening Dynamics. Two independent parameters govern the isothermal ripening experiments: the ripening time $t_{\mathrm{r}}$ and the temperature $T$. Scaling was attempted here with respect to these two parameters and with respect of the SEC moduli, $G_{\infty}^{\prime}(\omega, T)$ and $G_{\infty}^{\prime \prime}(\omega, T)$. We began with the rescaling of isothermal $G^{\prime}, G^{\prime \prime}\left(\omega, t_{\mathrm{r}}, T\right)$ data at discrete "ripening times" $t_{\mathrm{r}, 1}, t_{\mathrm{r}, 2}$, and $t_{\mathrm{r}, 3}$. Surprisingly, the shape of $G^{\prime}$ and $G^{\prime \prime}$ curves did not change significantly during 

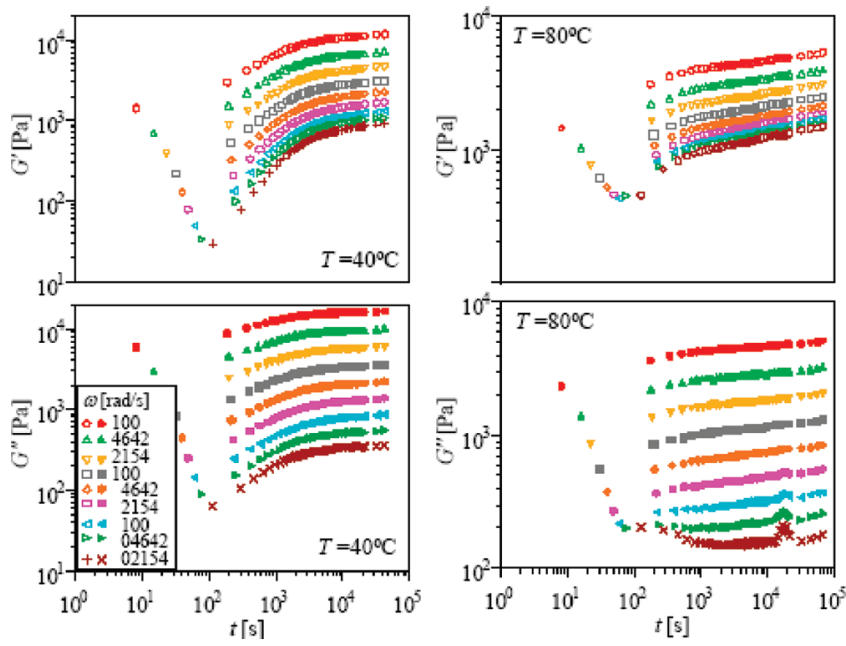

Figure 5. Isothermal ripening experiments for a freshly mixed $8 \mathrm{wt} \%$ clay/polymer nanocomposite at $40{ }^{\circ} \mathrm{C}$ (left) and $80{ }^{\circ} \mathrm{C}$ (right). Timeresolved dynamic modulus data strings belong to constant frequencies $\omega=100,46.42,21.54,10.0,4.642,2.154,1.00,0.4642$, and $0.2154 \mathrm{rad} / \mathrm{s}$, the top string belonging to the highest and the bottom string belonging to the lowest frequency. Corresponding measurements at 26, 50, 60, and $70{ }^{\circ} \mathrm{C}$ gave similar looking results.
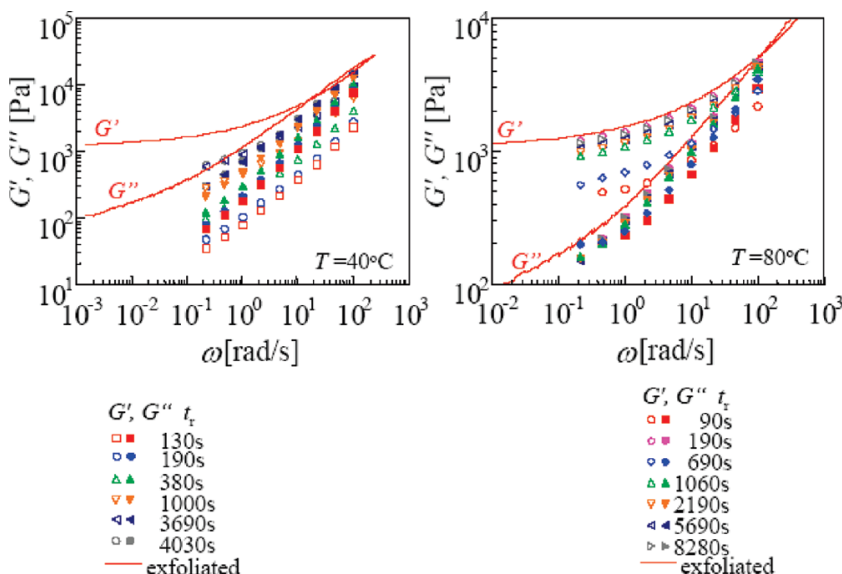

$G^{\prime}, G^{\prime \prime} t_{s}$

$90 \mathrm{~s}$

- $190 \mathrm{~s}$

6905

$\nabla 21905$

$\checkmark 4560 \mathrm{~s}$

$\rightarrow$ 8280s

Figure 6. Evolution of dynamic moduli during isothermal ripening. The snapshot data at discrete ripening times were generated by interpolation of the data in the previous figure. The SEC master curves at $T=40^{\circ} \mathrm{C}$ and $T=80^{\circ} \mathrm{C}$ are shown in comparison (continuous lines). The plot shows only $25 \%$ of the data so that individual curves can be recognized.

isothermal ripening so that they could be collapsed onto the corresponding SEC curve (see Figure 7a)

$$
\begin{aligned}
& G_{\infty}^{\prime}(\omega, T)=b_{\mathrm{r}} G^{\prime}\left(\omega a_{\mathrm{r}}, t_{\mathrm{r}}, T\right) ; \\
& G_{\infty}^{\prime \prime}(\omega, T)=b_{\mathrm{r}} G^{\prime \prime}\left(\omega a_{\mathrm{r}}, t_{\mathrm{r}}, T\right)
\end{aligned}
$$

The scaling of $G^{\prime}, G^{\prime \prime}$ data is based on relaxation processes that are fairly slow, as observed in oscillatory shear at low or intermediate frequencies, since this study is mostly concerned with the long-range connectivity of the evolving network structure as represented by the slow modes. Highfrequency data follow other scaling relations, which are not pursued here. The low-frequency scaling defines the horizontal and vertical "ripening shift" factors, $a_{\mathrm{r}}=a_{\mathrm{r}}\left(t_{\mathrm{r}} ; \infty, T\right)$ and $b_{\mathrm{r}}=b_{\mathrm{r}}\left(t_{\mathrm{r}} ; \infty, T\right)$, respectively. The bracket $\left(t_{\mathrm{r}} ; \infty\right)$ denotes the "ripening shift" between an intermediate state, $t_{\mathrm{r}}$, and SEC, $t_{\mathrm{r}} \rightarrow \infty$. (a)

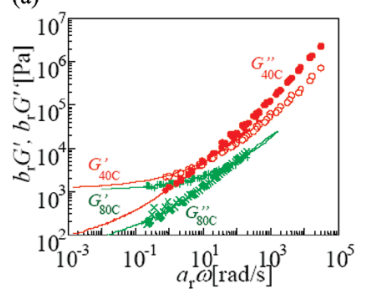

(b)

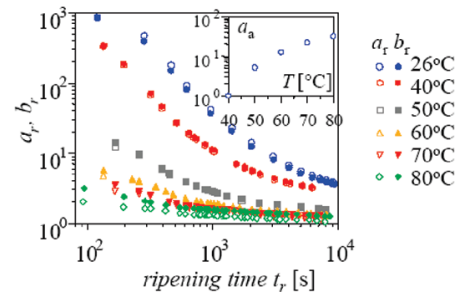

Figure 7. Ripening of $8 \mathrm{wt} \%$ clay in CTPB: (a) $G^{\prime}, G^{\prime \prime}$ data of Figure 5 after interpolating according to the time-resolved rheometry procedure $^{27}$ and shifting onto the SEC master curve (lines). For comparison, the corresponding $G^{\prime}, G^{\prime \prime}$ data of ripening at $80{ }^{\circ} \mathrm{C}$ is added. The difference between the two data sets $\left(40^{\circ} \mathrm{C}\right.$ vs $\left.80^{\circ} \mathrm{C}\right)$ is due to the temperature dependence as shown in Figure 4. (b) Shift factors for horizontal and vertical shift with SEC as reference, open symbols for $a_{\mathrm{r}}$ and filled symbols for $b_{\mathrm{r}}$. Most of the corresponding data points sit on top of each other. The magnitude of $\left(a_{\mathrm{r}}-1\right)$ or $\left(b_{\mathrm{r}}-1\right)$ is a measure of the "distance" from the SEC. Inset: the acceleration shift factor, $a_{\mathrm{a}}$, that merges all curves into the single shift function of Figure 8.

Interesting for this study is the specific physical meaning of the shift functions. The horizontal shift defines the reduction of the characteristic time $\lambda_{\mathrm{c}}\left(t_{\mathrm{r}}\right) / \lambda_{\mathrm{c}, \infty}$, and the vertical shift defines the increase of the characteristic modulus, $G_{\mathrm{c}}\left(t_{\mathrm{r}}\right) / G_{\mathrm{c}, \infty}$

$$
a_{\mathrm{r}}\left(t_{\mathrm{r}} ; \infty ; T\right)=\frac{\lambda_{\mathrm{c}}\left(t_{\mathrm{r}}, T\right)}{\lambda_{\mathrm{c}, \infty}(T)} ; \quad 1 / b_{\mathrm{r}}\left(t_{\mathrm{r}} ; \infty ; T\right)=\frac{G_{\mathrm{c}}\left(t_{\mathrm{r}}, T\right)}{G_{\mathrm{c}, \infty}(T)}
$$

Both time shift $a_{\mathrm{r}}$ and the modulus shift $b_{\mathrm{r}}$ approach unity at very long times when the structure has ripened to its SEC state. The ripening "scaling ratio"

$$
n_{\mathrm{r}}=\left(\log b_{\mathrm{r}}\right) /\left(\log a_{\mathrm{r}}\right)
$$

defines the slope of the rescaling of the dynamic data $\left(n_{\mathrm{r}}=1\right.$ for shift at $45^{\circ}$ ).

The most surprising finding from our experiments is also shown in Figure 7b: The two ripening shift functions $a_{\mathrm{r}}$ and $b_{\mathrm{r}}$ were found to be equal (scaling ratio $n_{\mathrm{r}} \approx 1$ ) for ripening experiments at elevated temperatures $(T=40,50,60$, and $70^{\circ} \mathrm{C}$ ). Only for ripening at 26 and $80^{\circ} \mathrm{C}, a_{\mathrm{r}}$ and $b_{\mathrm{r}}$ deviate slightly from each other. This means that the product of characteristic modulus and relaxation time

$$
G_{\mathrm{c}}\left(t_{\mathrm{r}}, T\right) \lambda_{\mathrm{c}}\left(t_{\mathrm{r}}, T\right) \approx G_{\mathrm{c}, \infty}(T) \lambda_{\mathrm{c}, \infty}(T)
$$

stays constant throughout most of the ripening process $\left(G \lambda=360 \mathrm{~Pa} \mathrm{~s}\right.$ at $\left.T=40^{\circ} \mathrm{C}\right)$.

\section{Heuristic Model of Ripening Dynamics}

The ripening dynamics accelerates at elevated temperatures but the shape of the ripening shift factors seems to be temperature invariant (see Figure 7b). A horizontal shift along the ripening time axis is sufficient to merge; in a reasonably good approximation, all relaxation data into a single master curve for the entire ripening dynamics (Figure 8$), f\left(a_{\mathrm{a}} t_{\mathrm{r}}, T\right)=b_{\mathrm{r}}\left(t_{\mathrm{r}} ; \infty ; T_{\text {ref }}\right)=a_{\mathrm{r}}\left(t_{\mathrm{r}} ; \infty\right.$; $\left.T_{\text {ref }}\right)$. The ripening acceleration factor, $a_{\mathrm{a}}$, as shown in Figure $7 \mathrm{~b}$, inset, can be expressed as Taylor expansion $a_{\mathrm{a}}\left(T ; T_{\text {ref }}\right)=1+$ $\alpha\left(T-T_{\text {ref }}\right)-\beta\left(T-T_{\text {ref }}\right)^{2}$. Above $T=40^{\circ} \mathrm{C}$, the quadratic term of the acceleration factor is small, $\alpha\left(T-T_{\text {ref }}\right) \ll \beta\left(T-T_{\text {ref }}\right)^{2}$, within the experimental region. The ripening function, as a quantitative measure of the distance from SEC, decays rapidly at first and continues very slowly. This suggests a sequence of two distinctly different mechanisms which may be expressed in an empirical ripening function

$$
f\left(t_{\mathrm{r}}, T\right)-1=\left(f_{0}-1\right)\left\{\left(\frac{t_{\mathrm{r}, 0}}{t_{\mathrm{r}}}\right)^{-2}+\left(\frac{t_{\mathrm{r}, 0}}{t_{\mathrm{r}}}\right)^{-1 / 2}\right\}
$$


with parameters $f_{0}=2.6$ and $t_{\mathrm{r}, 0}=54.5 \mathrm{~s}$ (at $\left.T=40{ }^{\circ} \mathrm{C}\right)$. The overlay of this function together with the data is shown in Figure 8. All experiments condense into a single scaling relation. The ripening function decays to value $1, \lim _{t_{\mathrm{r}} \rightarrow \infty} f\left(a_{\mathrm{a}} t_{\mathrm{r}}, T\right)=1$, at long times because of SEC being the reference state.

\section{Discussion}

One of the objectives of this study is the formation of a nanocomposite that is random with respect to its clay orientation. This needs to be emphasized since, as separate rheological experiments in preparation of this study have shown, shearinduced clay orientation is not able to relax back into randomness even after extremely long annealing times. Rheologically, this expresses itself in a low modulus due to a reduced internal connectivity of the oriented clay-polymer network. Data will depend on specific stain histories. To avoid this, it is important that we start out with a sample of random orientation and preserve randomness during the mixing of clay into the polymer. We take advantage of the fact that the clay by itself consists of aggregates in which clay particles (stacks of aligned day sheets) are randomly assembled and that randomness is protected as long as the aggregate state is preserved. Keeping this in mind, we mixed clay and polymer under mild shearing conditions without breaking the clay aggregates. The mixing was distributive but at low stress. Unbroken aggregates are noticeable by the opaque appearance of the nanocomposite sample after mixing. Within an hour after mixing, the sample becomes optically clear due to the polymer-induced breaking of the clay aggregates into the small clay particles. Macroscopic randomness is maintained at these early ripening stages since the randomly oriented clay particles are still assembled as stacks of clay sheets. This provides the random initial condition for our experimental study of the structural ripening. Exfoliation times are orders of magnitude larger than the breakup time of aggregates in the presence of endfunctionalized polymer. The time-resolved rheological experiments involve only small shear strains so that the randomness of the clay sheets is maintained. All results of this study refer to randomly oriented clay structures. Oriented clay structures will be studied separately.

A uniform representation of ripening at all temperatures is possible because the material assumes a stable end-state which can serve as reference for the structure development. As an alternative, we could have rescaled the experimental observations with the critical gel state ${ }^{32}$ as has been done before for characterizing materials near the gel point. ${ }^{10,33}$ However, physical gels or soft glasses exhibit liquid-to-solid transition states that are not unique. Their critical gel state depends on the experimental path $^{34,35}$ and possibly on the connectivity of trapped intermediate states. This problem of path dependence is avoided by rescaling the ripening dynamics with the stable end state (SEC) as defined above.

The shift factors of Figure $7 \mathrm{~b}$ express the distance of the ripening structure from SEC. We could easily reach SEC at temperatures above $80{ }^{\circ} \mathrm{C}$ but only could see how closely the structure approaches SEC when ripening at 70, 60, 50, 40, and $26{ }^{\circ} \mathrm{C}$. We also performed an extremely long experiment at $40{ }^{\circ} \mathrm{C}$; however, it could not reach SEC within 3 days. We ascribe this to large energy barriers at temperatures below $50^{\circ} \mathrm{C}$. The shifting of entire modulus curves into a master curve requires that the shape of all curves remains constant during the ripening process. This is the case for relaxation modes throughout an intermediate frequency range. More experiments are needed to quantify the frequency limits of this behavior.

For colloidal nanocomposites, the diffusion of polymer molecules into the clay galleries and, at later stages, diffusion of exfoliated clay leaves are known to be governed by several

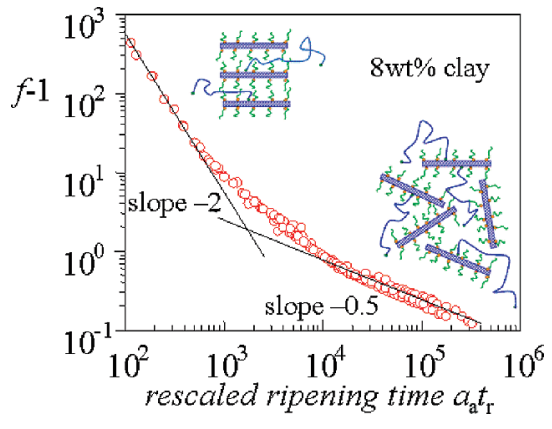

Figure 8. Ripening function as defined in eq 7 is dominated by two processes: fast intercalation and slow exfoliation. The reference temperature is $T_{\text {ref }}=40^{\circ} \mathrm{C}$.

competing processes and phase behavior. ${ }^{14-16,36}$ Cocard et al. found the shear modulus to continue to evolve and a stable state was not reached after having passed the gel point of gelling Laponite clay suspensions. ${ }^{13}$ The loss modulus of the gel increased at low frequencies, indicating the presence of slow relaxation processes. This complexity makes it even more surprising that a single function is able to describe the overall change in rheology. The specific shape of the scaling function of Figure 8 is sample specific. However, in spite of the fundamental difference of these two structuring processes, suspension-type structure followed by an exfoliated leaf structure, the property $G_{\mathrm{c}}\left(t_{\mathrm{r}}\right)-$ $\lambda_{\mathrm{c}}\left(t_{\mathrm{r}}\right)=$ constant is found for the entire structure evolution beyond the gel point. This indicates that $G_{\mathrm{c}} \lambda_{\mathrm{c}}=$ constant might be more widely found with other complex materials during structural ripening. Many more experiments on other physical gels and soft glasses will be required to define the range of validity. The experimentally found scaling ratio value of unity, $n_{\mathrm{r}}=1$, is so specific that it will be interesting to see whether other physical gels exhibit the scaling property but require another $n_{\mathrm{r}}$ value.

The property $G_{\mathrm{c}}\left(t_{\mathrm{r}}\right) \lambda_{\mathrm{c}}\left(t_{\mathrm{r}}\right)=$ constant has consequences for the modeling of viscoelasticity of physical gels and soft glasses. These need to be explored further. An example is the complex viscosity. The product $G \lambda$ is property of a solid but has the dimension of a viscosity. For $n_{\mathrm{r}}=1$, the complex viscosity, $\eta^{*}(\omega)$ or $\eta^{*}\left(G^{*}\right)$, shifts horizontally during the ripening process:

$$
\begin{gathered}
\frac{b_{\mathrm{r}}}{a_{\mathrm{r}}} \eta^{*}\left(a_{\mathrm{r}} \omega, t_{r}, T\right) \approx \eta_{\infty}^{*}(\omega, T) \text { or } \frac{b_{\mathrm{r}}}{a_{\mathrm{r}}} \eta^{*}\left(b_{\mathrm{r}} G^{*}, t_{\mathrm{r}}, T\right) \\
\approx \eta_{\infty}^{*}\left(G^{*}, T\right)
\end{gathered}
$$

This is shown in Figure 9 for four ripening states and the shift onto SEC. $\eta^{*}$ is finite for liquids and solids. Different from the steady shear viscosity, $\eta^{*}$ does not diverge at the liquid-to-solid transition (except for its low- $G^{*}$ limit or its low- $\omega$ limit). $G^{*}$ is chosen here as abscissa since it magnifies differences between material states. $^{37,38}$

The frequency-dependent moduli shift without changing their shape. That means that the relaxation time spectrum for an early structural state and its corresponding relaxation time spectrum of the final structural state, when written in discrete form

$$
\begin{aligned}
G\left(t, t_{\mathrm{r}}, T\right) & =\sum g_{i}\left(t_{\mathrm{r}}, T\right) \mathrm{e}^{-t / \lambda_{i}\left(t_{\mathrm{r}}, T\right)}, \\
G_{\infty}(t, T) & =\sum g_{i, \infty}(T) \mathrm{e}^{-t / \lambda_{i, \infty}(T)}
\end{aligned}
$$

are related through

$$
\begin{aligned}
& \lambda_{i}\left(t_{\mathrm{r}}, T\right)=a_{\mathrm{r}}\left(t_{\mathrm{r}} ; \infty ; T\right) \lambda_{i, \infty}(T) ; \\
& g_{i}\left(t_{\mathrm{r}}, T\right)=g_{i, \infty}(T) / b_{\mathrm{r}}\left(t_{\mathrm{r}} ; \infty ; T\right)
\end{aligned}
$$

It should be noted that this shifting property belongs to the observed frequency window and that there are upper and lower 


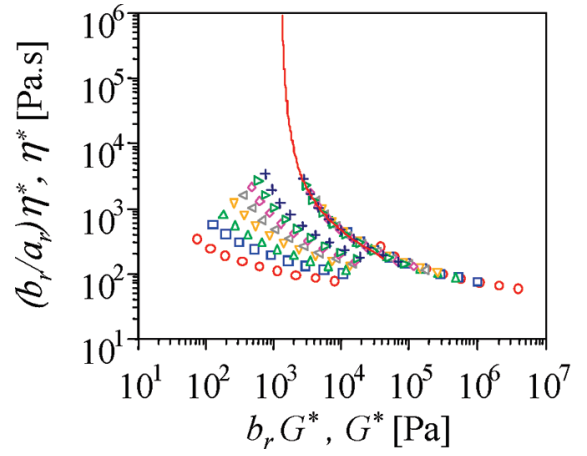

time $t_{\mathrm{r}}$

- $116 \mathrm{~s}$

295s

$\triangle 437 \mathrm{~s}$

$\nabla \quad 676 \mathrm{~s}$

$\triangleleft \quad 1000 \mathrm{~s}$

- $1603 \mathrm{~s}$

$\triangle \quad 2541 \mathrm{~s}$

$+\quad 4898 \mathrm{~s}$

SEC

Figure 9. Complex viscosity at increasing ripening states and merged into a master curve at the reference temperature. The shift is horizontal because of an $n_{\mathrm{r}}$ value of 1 . The reference temperature is $T_{\text {ref }}=40^{\circ} \mathrm{C}$. For comparison, the viscosity of the pure matrix fluid is $22 \mathrm{~Pa}$ s at $T_{\text {ref }}=$ $40{ }^{\circ} \mathrm{C}$.

limits of the proposed dynamics, which need to be explored further.

\section{Conclusions}

The sample preparation method is ideally suited for preparing nanocomposites of random clay orientation. The random clay/ polymer system (with 8 wt \% clay) reached its most stable structure independently of the experimental path (using three different protocols), which is a prerequisite for using it as reference. The liquid-to-solid transition (gel point), which can be recognized by its power-law relaxation modulus, $G(t)=S t^{-n}$, occurs very quickly after mixing the clay into the polymer. The consecutive ripening in the solid state evolves in a stunningly regular pattern with distinct properties that have never been observed before to our knowledge. Surprisingly, the product of characteristic modulus and time, $G_{\mathrm{c}} \lambda_{\mathrm{c}}$, stays constant throughout the ripening process while both properties by themselves undergo large change. All low-frequency experiments collapse into a single scaling relation with a sequence of two pronounced power-law regions: a fast ripening process $\left(\sim t_{\mathrm{r}}{ }^{2}\right)$ followed by slow ripening $\left(\sim t_{\mathrm{r}}^{-1 / 2}\right)$. The ripening time, $t_{\mathrm{r}}$, is the independent parameter, which defines the state of ripening and projects the time necessary to reach the stable exfoliated clay (SEC) state. The experimental protocol of this study is ideally suited for characterizing materials with time-dependent structure in general and for exploring the range of validity of the new findings for a wider class of colloidal solids.

Acknowledgment. H.H.W. acknowledges the support of NSF CBET 0651888. The study was supported by National Natural Science Foundation of China (Grants 20774041, 20825416, and 20904020), the China Postdoctoral Science Foundation, and Jiangsu Planned Projects for Postdoctoral Research Funds.

\section{References and Notes}

(1) Stradner, A.; Sedgwick, H.; Cardinaux, F.; Poon, W. C. K.; Egelhaaf, S. U.; Schurtenberger, P. Nature 2004, 432, 492.

(2) Sciortino, F.; Mossa, S.; Zaccarelli, E.; Tartaglia, P. Phys. Rev. Lett. 2004, 93, 4.

(3) Dijkstra, M.; Hansen, J. P.; Madden, P. A. Phys. Rev. Lett. 1995, $75,2236$.

(4) Ovarlez, G.; Coussot, P. Phys. Rev. E 2007, 76, 011406.

(5) Fleming, G. R.; Ratner, M. A. Phys. Today 2008, 61, 28.

(6) King, H. E.; Milner, S. T.; Lin, M. Y.; Singh, J. P.; Mason, T. G. Phys. Rev. E 2007, 75, 20.

(7) Giannelis, E. P.; Krishnamoorti, R.; Manias, E. Adv. Polym. Sci. 1999, $138,107$.

(8) Winter, H. H.; Mours, M. Adv. Polym. Sci. 1997, 134, 165.

(9) Nijenhuis, K. T. Adv. Polym. Sci. 1997, 130, 1.

(10) Adolf, D.; Martin, J. E. Macromolecules 1990, 23, 3700.

(11) Kakiuchi, M.; Aoki, Y.; Watanabe, H.; Osaki, K. Macromolecules 2001, 34, 2987.

(12) Trappe, V.; Weitz, D. A. Phys. Rev. Lett. 2000, 85, 449.

(13) Cocard, S.; Tassin, J. F.; Nicolai, T. J. Rheol. 2000, 44, 585.

(14) Mourchid, A.; Delville, A.; Lambard, J.; Lecolier, E.; Levitz, P. Langmuir 1995, 11, 1942.

(15) Mourchid, A.; Delville, A.; Levitz, P. Faraday Discuss. 1995, 275.

(16) Mourchid, A.; Levitz, P. Phys. Rev. E 1998, 57, R4887.

(17) Awasthi, V.; Joshi, Y. M. Soft Matter 2009, 5, 4991.

(18) Shukla, A.; Joshi, Y. M. Chem. Eng. Sci. 2009, 64, 4668.

(19) Chen, T. H.; Zhu, J. J.; Li, B. H.; Guo, S. Y.; Yuan, Z. Y.; Sun, P. C.; Ding, D. T.; Shi, A. C. Macromolecules 2005, 38, 4030

(20) Zhu, J. J.; Wang, X. L.; Tao, F. F.; Xue, G.; Chen, T. H.; Sun, P. C.; Jin, Q. H.; Ding, D. T. Polymer 2007, 48, 7590.

(21) Wang, X. L.; Gao, Y.; Mao, K. M.; Xue, G.; Chen, T. H.; Zhu, J. J.; Li, B. H.; Sun, P. C.; Jin, Q. H.; Ding, D. T.; Shi, A. C. Macromolecules 2006, 39, 6653.

(22) Wang, X. L.; Tao, F. F.; Xue, G.; Zhu, J. J.; Chen, T. H.; Sun, P. C.; Winter, H. H.; Shi, A. C. Macromol. Mater. Eng. 2009, 294, 190.

(23) Ray, S. S.; Okamoto, M. Prog. Polym. Sci. 2003, 28, 1539.

(24) Pinnavaia, T. J.; Beale, G. W. Polymer-Clay Nanocomposites; Wiley: New York, 2000.

(25) Balazs, A. C.; Singh, C.; Zhulina, E. Macromolecules 1998, 31, 8370.

(26) Sinsawat, A.; Anderson, K. L.; Vaia, R. A.; Farmer, B. L. J. Polym. Sci., Part B: Polym. Phys. 2003, 41, 3272.

(27) Mours, M.; Winter, H. H. Rheol. Acta 1994, 33, 385.

(28) Winter, H. H.; Chambon, F. J. Rheol. 1986, 30, 367.

(29) Winter, H. H.; Mours, M. Rheol. Acta 2006, 45, 331.

(30) Ferry, J. D. Viscoelastic Properties of Polymers, 3rd ed.; John Wiley \& Sons: New York, 1980.

(31) Holly, E. E.; Venkataraman, S. K.; Chambon, F.; Winter, H. H. J. Non-Newtonian Fluid Mech. 1988, 27, 17.

(32) Vilgis, T. A.; Winter, H. H. Colloid Polym. Sci. 1988, 266, 494.

(33) DeRosa, M. E.; Mours, M.; Winter, H. H. Polym. Gels Networks $1997,5,69$

(34) Horst, R. H.; Winter, H. H. Macromolecules 2000, 33, 130.

(35) Horst, R. H.; Winter, H. H. Macromolecules 2000, 33, 7538.

(36) Bousmina, M. Macromolecules 2006, 39, 4259.

(37) Booij, H. C.; Palmen, J. H. M. In Moldenaers, P., Keunings, R., Eds.; Theoretical and Practical Rheology; Elsevier: Brussels, 1992; p 321.

(38) Winter, H. H. Rheol. Acta 2009, 48, 241. 